\title{
Gestión del conocimiento y gestión de la información
}

Carlota Bustelo Ruesta

Raquel Amarilla Iglesias

INFORAREA S.L.

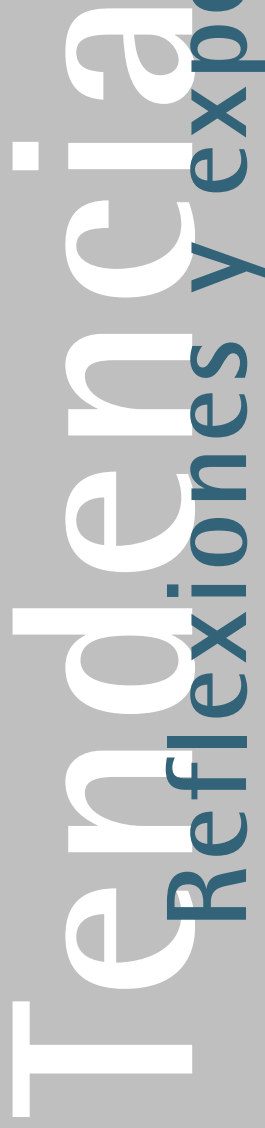

Resumen

La Gestión del conocimiento y todo lo que tiene que ver con ella, ha irrumpido con fuerza últimamente en el panorama de la gestión de todo tipo de organizaciones, sobre todo gracias al auge que las Nuevas Tecnologías están experimentando y su repercusión en el desarrollo de la denominada Sociedad de la Información. Aunque surge dentro del campo empresarial, cualquier tipo de organización puede guiarse por esta nueva filosofía. El presente artículo trata, pues, de ofrecer una definición aproximativa de lo que se entiende Gestión del conocimiento y de los elementos de que se compone, señalando qué tipos de iniciativas prácticas se están desarrollando al respecto. Además, se trata de diferenciar este concepto de la Gestión de Información, diferencia que viene dada por la contraposición de dos conceptos fundamentales: conocimiento tácito y conocimiento explícito.

\section{Palabras clave}

Gestión del Conocimiento / Gestión de la Información / Capital Intelectual / Internet / Sociedad de la Información

\section{Introducción}

Desde hace algún tiempo, se habla mucho de Gestión del Conocimiento, presentándola como una tendencia de futuro imprescindible para encarar los retos de la Sociedad de la Información. Los discursos teóricos se suceden en distintos foros convenciéndonos de ello, mientras que las experiencias prácticas que se acogen a esta denominación nos sorprenden por la sensación de que "no hay nada nuevo bajo el sol", sobre todo para aquellos que trabajamos en la gestión de la información. Hay quien afirma que esta forma de gestión no es más que una moda, que no ha aportado nada radicalmente nuevo, pero lo cierto es que las estrategias comerciales de vendedores de software y consul- tores de todo tipo han dado un vuelco importante y casi todos "venden" gestión del conocimiento, al tiempo que las grandes empresas están implantando programas de gestión del conocimiento. Este artículo pretende explicar lo que, desde nuestra perspectiva, tiene de novedad la gestión del conocimiento y cual es su relación con la gestión de la información, para terminar reflexionando sobre la gestión del conocimiento fuera del ámbito estrictamente empresarial.

\section{¿Qué es la Gestión del Conocimiento?}

\section{Un intento de definición}

Huyendo de la repetición de las definiciones de los grandes "gurus" creemos que la gestión del conocimiento es todo el conjunto de actividades realizadas con el fin de utilizar, compartir y desarrollar los conocimientos de una organización y de los individuos que en ella trabajan, encaminándolos a la mejor consecución de sus objetivos.

Esta definición nos abre un marco muy amplio y, además, requiere del desarrollo de una serie de conceptos teóricos que nos permitan entender cual es la materia a gestionar.

\section{Algunos conceptos teóricos}

- El primer concepto a aclarar es qué se entiende por conocimiento. Sin entrar en profundidades filosóficas, que darían lugar a mucho más que un artículo, el conocimiento en una organización se produce cuando un individuo de la misma hace uso de lo que sabe y de la información que tiene disponible para la resolución de un problema o el desarrollo de un proyecto. Los expertos distinguen entre dos tipos de conocimientos: Conocimiento explícito y tácito. El primero es el que dentro de la organización tiene establecidas las fórmulas por las cuales se puede trasmitir a otras personas (un ejemplo de gestión del conocimiento explícito se ha dado siempre en la comunidad científica, que comparte con otros científicos los resultados de sus investigaciones). Por el contrario, el conocimiento tácito, es aquel que toda organización tiene, pero que no queda plasmado ni registrado en lugar alguno, estando totalmente ligado al grupo de personas que componen la organización en cada momento. (Fig. I)

- Si se profundiza en la teoría que da soporte a la gestión del conocimiento, se llegará pronto a la conclusión de que el conocimiento es un recurso 
importante para las organizaciones empresariales. Esto explicaría que empresas como Microsoft o Dell se coticen en la bolsa multiplicando varias veces su valor real contable. El conocimiento entraría en la categoría de los activos intangibles que muchos estudiosos tratan de cuantificar para medir el denominado capital intelectual .Siguiendo a Edvinsson y Malone ', la mejor forma de entender el papel del capital intelectual es compararlo con un organismo vivo, por ejemplo un árbol. Lo que se describe en los organigramas, los informes,...es el tronco, las ramas y las hojas. Pero, aunque esta sea la parte del árbol visible, no lo es todo. La mitad al menos está bajo tierra, son las raíces. Éstas, en nuestra metáfora, constituirían el capital intelectual de la organización: el estudio de las raíces de valor de la empresa. Así, los tipos de valor a tener en cuenta a la hora de medirlos, se podrían dividir en tres clases:

- Capital humano: lo constituyen los conocimientos, habilidades, experiencias,... de los empleados individuales de la organización

- Capital estructural: son los equipos, programas, bases de datos, estructura organizativa,...y todo lo que forma parte de la capacidad organizacional de una empresa ("todo lo que se queda en la oficina cuando los empleados se van a su casa")

- Capital cliente 2: se trata del fruto del desarrollo de relaciones con los clientes claves de una organización

\section{El entorno que la hace posible}

Otro aspecto que choca a la hora de abordar el tema de la Gestión del conocimiento es ipor qué ahora? Hay párrafos sorprendentes en los escritos de los primeros economistas en los que estas ideas se exponen con bastante claridad, como es el caso de los escritos del economista inglés Alfred Marshall 3. Se puede decir que él es el primer autor en hacer referencia a la importancia del conocimiento en la gestión y, por ende, en la economía. En su obra Principios de Economía publicada en 1890 dice, al hablar de los agentes de la producción, que "el conocimiento es nuestra máquina de producción más potente" y que "la organización ayuda al conocimiento". Sin embargo, es el desarrollo tecnológico actual el que permite que podamos vislumbrar nuevos métodos de gestión que supongan un cambio profundo. Es decir no se entiende la "moda" de la Gestión del Conocimiento sin Internet y el desarrollo de las tecnologías de la información.

La evolución del desarrollo tecnológico ha sido tan importante en los últimos años, que ha supuesto muchos cambios profundos en la manera de trabajar, de hacer negocios y en la propia sociedad. Muchos de estos cambios están todavía en evolución y necesitan de un corpus teórico en el que asentarse. En este sentido, podríamos decir que la "gestión del conocimiento" es la teoría de gestión que responde a la adaptación de las últimas innovaciones tecnoló-

\begin{tabular}{|c|c|c|}
\hline \multicolumn{3}{|c|}{ Figura 1. Fuente Sáiz Carrasco, Angel. (1999) } \\
\hline \multicolumn{2}{|r|}{$\begin{array}{l}\text { Información } \\
\text { - Esadísticas y datos } \\
\text { - Teorías } \\
\text { - Red de contactos }\end{array}$} & \multirow{2}{*}{$\begin{array}{l}\text { Cultura } \\
\text { - Valores } \\
\text { - Intuicuiones } \\
\text { - Opiniones } \\
\text { Capacidades } \\
\text { - Experiencia personal } \\
\text { - Grupos de expertos }\end{array}$} \\
\hline \multirow[t]{2}{*}{ SABER CÓMO } & $\begin{array}{l}\text { Procedimientos } \\
\text { - Políticas internas } \\
\text { - Manual del proceso } \\
\text { - Experiencia y antecedentes }\end{array}$ & \\
\hline & $\begin{array}{l}\text { CONOCIMIENTO } \\
\text { EXPLÍCITO }\end{array}$ & $\begin{array}{c}\text { CONOCIMIENTO } \\
\text { TÁCITO }\end{array}$ \\
\hline
\end{tabular}

gicas en el tratamiento de la información y las telecomunicaciones.

Los economistas hablan de la nueva era del Conocimiento o la Sociedad de la información, en la que la correlación de fuerzas entre los recursos en los que se basa la economía mundial (tierra, capital trabajo y conocimiento) cambia radicalmente.

Para ilustrar este cambio se utiliza habitualmente un esquema simple pero muy ilustrativo que reproducimos aquí. (Figura 2)

Figura 2. Fuente Gorey y Dorat (1996) y Bueno(1999)

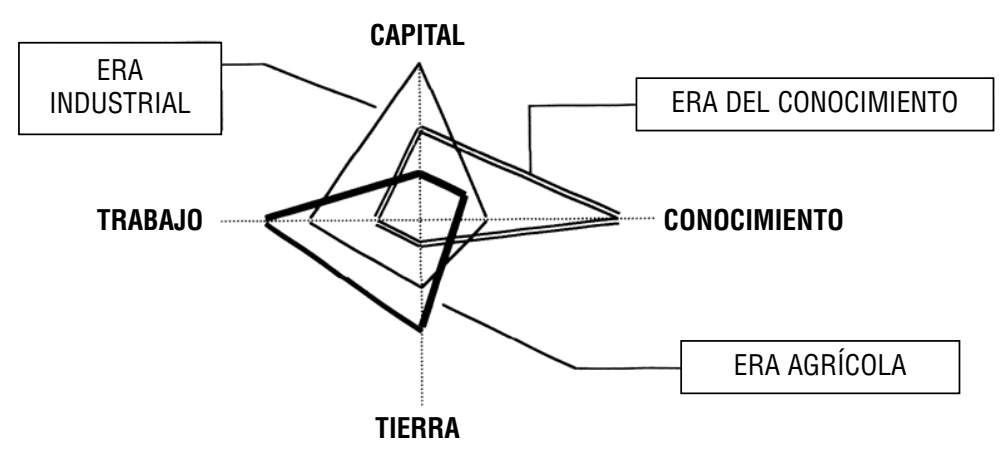

Las aplicaciones prácticas

Como hemos visto hasta ahora, la Gestión del Conocimiento es más una filosofía de gestión, que realmente unas técnicas o unos medios específicos que se puedan aplicar en cualquier entorno. Por eso debemos tener en cuenta que la gestión del conocimiento no se puede implantar en un corto espacio de tiempo y esta es la razón de que las experiencias prácticas que nos muestran nos "sepan a poco". En realidad lo que nos muestran son pequeñas iniciativas que se encaminan a facilitar la gestión del conocimiento en una organización (implantación de herramientas de software, establecimiento de sistemas de debate, sistema de recompensas por aportación 
de ideas, etc) o bien políticas de incrementar la competencia de los trabajadores (programas de adquisición de ordenadores para casa, programas de formación, etc.) o incluso intentos de medir el "conocimiento" dentro de la organización (indicadores que empiezan a aparecer en las memorias anuales de las empresas).

En cualquier caso, podemos distinguir tres frentes en los que se desarrollan las iniciativas prácticas:

- En la gestión de la información. La tendencia es a implantar sistemas que permitan que la información que tiene la organización y los individuos que la componen puedan ser compartida por todos. En este sentido, se está desarrollando mucho la elaboración de "mapas documentales" y de "conocimientos" en los que se representa y da acceso de manera gráfica a toda la tipología documental que se produce o maneja en una organización, de modo que todo el mundo sabe qué hace el resto de departamentos o personas de la misma. Este tipo de prácticas, suele implantarse en un entorno de Intranet, con el fin de darle la máxima utilidad y accesibilidad.

- En la gestión de recursos humanos. Para que las ideas fluyan y los individuos se sientan motivados a aportar sus conocimientos a la organización hay que establecer unas políticas de desarrollo de recursos humanos, que permitan que esto sea posible. Esto se logra a través de distintos medios, como por ejemplo la formación continua del personal - la celebración de encuentros o "communities" en los que distintos grupos de personas comparten "su conocimiento".

- En la medición de los activos intangibles. Las organizaciones tratan de medirlos para demostrar su potencial y su capacidad de enfrentar el futuro con éxito; así como para demostrar su crecimiento a lo largo del tiempo. En general, se trata de atribuir un valor contable a los recursos que resultan intangibles por el momento.

Para llegar a conseguir una verdadera "gestión del conocimiento" los tres frentes deben confluir y el avance no es tan rápido como nos sugiere la rapidez con la que se suceden los cambios tecnológicos. Entre otras razones, todas estas iniciativas pueden abordarse en un clima de bonanza económica dentro de la organización, pues aunque a la larga una mejor gestión debe suponer beneficios económicos, los primeros pasos exigen inversiones no siempre justificables.

Por lo tanto no debemos confundir la implantación de un programa de "gestión del conocimiento" con:

- La inversión en herramientas informáticas: en muchas organizaciones se ha pensado que abordar la implantación de un programa de gestión del conocimiento es sinónimo de adquirir una herramienta informática de última generación e implantar su uso a nivel corporativo. Esto es un gran error, porque la informática es un elemento fundamental para la gestión del conocimiento, pero no es el único.

- Incluir en los procedimientos de trabajo, la obligación de que las personas "documenten" lo que saben. Estas prácticas normalmente caen en desuso al poco de implantarse, pues las personas deben seguir cumpliendo con su trabajo y no se les ofrece nada por compartir su conocimiento.

\section{¿Es Gestión del conocimiento sólo Gestión de la Información?}

Retomando las ideas del punto anterior, uno de los frentes en los que se realiza la aproximación práctica a la gestión del conocimiento, es la gestión de la información. Por ello, no es extraño que entre los especialistas en gestión de la información surja la pregunta que da pie a este epígrafe. Siendo nuestra contestación negativa, intentaremos explicar cuales son sus diferencias partiendo de una definición de gestión de la información.

La gestión de la información se puede definir como el conjunto de actividades realizadas con el fin de controlar, almacenar y, posteriormente, recuperar adecuadamente la información producida, recibida o retenida por cualquier organización en el desarrollo de su actividades. En el centro de la gestión de la información se encuentra la gestión de la documentación (la información que queda plasmada en documentos) y que puede ser de tres tipos:

- Interna: hace referencia a aquella documentación generada o recibida por la organización en el ejercicio de sus funciones, es decir, son documentos que surgen de la actividad diaria de esa institución. En este grupo, encontramos tanto la documentación típicamente administrativa: contabilidad, correspondencia,..., como la documentación de gestión: informes, actas de reuniones, procedimientos de trabajo, y la documentación técnica que refleja la propia actividad de la organización.

- Externa: además de la documentación producida por la propia organización, ésta y las personas que trabajan en ella necesitan, a menudo, consultar y manejar fuentes de información externas: libros revistas, B.D., Internet

- Pública: es aquella documentación que la organización produce de cara al público, para comunicarles hechos, actividades, acontecimientos,...por ejemplo, las memorias, los catálogos de productos y servicios, la página web,...

Además de la gestión de la documentación existe, estrechamente relacionada con ella, la gestión de información repartida en bases de datos corporativas y aplicaciones informáticas, que no se conceptúan 
como documentos; pero que son una importante fuente de información registrada.

Desde nuestro punto de vista, sin una adecuada gestión de la información, es imposible llegar a la gestión del conocimiento. Las propuestas de la gestión del conocimiento representan un modelo de gestión que se basa en gran parte en gestionar adecuadamente la información. Es, por lo tanto, el paso previo que cualquier organización debería dar antes de tratar de implantar un sistema de gestión del conocimiento. Esta idea es la que quiere representar la figura 3.

Para nosotras, hay varios componentes que son necesarios para dar el salto de la gestión de la información a la gestión del conocimiento.

- En primer lugar, la gestión del conocimiento es un modelo de gestión de toda la organización, cosa que nunca ha sido la gestión la información. Dependiendo del estilo del empresario y de la organización, los sistemas de gestión de la información, cada vez más importantes a medida que las tecnologías ofrecían nuevas posibilidades, se adaptaban a los modelos de gestión existentes.

- Para gestionar el conocimiento hay que tener en cuenta que este no se produce sólo por la gestión de la información, sino que deben intervenir procesos y personas. En una organización puede existir un perfecto modelo de gestión de la información, pero si los individuos no lo utilizan es imposible que se cree conocimiento. Por esta razón otra de las tendencias muy involucradas en la definición de la gestión del conocimiento es la que proviene de la gestión de los recursos humanos. La gestión de la motivación, del talento, del trabajo en equipo y, sobre todo, la creación de un ambiente de trabajo que facilite la compartición de ideas, es una tarea a la que difícilmente se accede a través de la gestión de la información.

\section{¿La Gestión del Conocimiento sólo es posible en el ámbito empresarial?}

Desde luego, el nacimiento de la idea de la "gestión del conocimiento" parte de la realidad empresarial y, por lo tanto, lo que busca en última instancia es aumentar el beneficio económico de las empresas. Sin embargo, sus ideas pueden ser aplicables a entornos en los que no se busca el beneficio económico, sino el beneficio social o cultural. De la misma manera que anteriormente se han aplicado otras tendencias nacidas en el entorno de la empresa privada como la gestión de calidad, las técnicas de marketing o la planificación estratégica, la gestión del conocimiento tiene cabida en la administración pública, en instituciones culturales y en organizaciones $\sin$ ánimo de lucro.
Las ideas claves a aplicar son las siguientes:

- La gestión del conocimiento, permite realizar más eficazmente el trabajo encomendado a la organización.

- Mediante la gestión del conocimiento las organizaciones favorecen que el individuo se desarrolle en su trabajo aportando ideas, al mismo tiempo que evita la "fuga de conocimiento" cuando las personas abandonan la organización.

- La gestión de la información es imprescindible, pero sólo se convierte en conocimiento cuando los individuos la aplican para la resolución de un problema.

En este sentido, al mismo tiempo que en la empresa privada se han desarrollado iniciativas para la gestión del conocimiento, en las administraciones públicas de nuestro entorno podemos encontrar iniciativas paralelas que, quizás sin haber utilizado la misma denominación ni plantearse los mismos objetivos, se basan en idénticos principios. Entre ellas podemos encontrar el esfuerzo en la gestión de la información para la toma de decisiones, o la implantación de sistemas que permiten explotar el conocimiento de los "clientes".

\section{Figura 3: Elaboración propia}

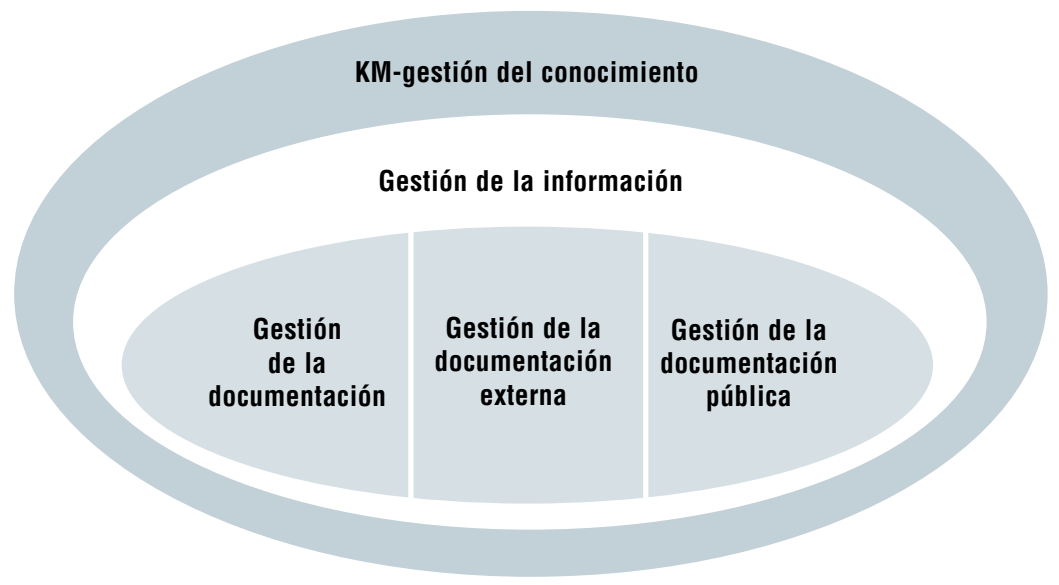

\section{Conclusión: ...y en el futuro?}

En estos tiempos de cambios tan vertiginosos impulsados por la innovación tecnológica, es muy arriesgado formular opciones de futuro. Aún así, creemos que la tendencia marcada por las teorías de la "gestión del conocimiento" es imparable. La sociedad de la información requiere de organizaciones, tanto públicas como privadas, que se adapten a los nuevos baremos y que sean capaces de aprovechar, en su propio beneficio, las tecnologías de la información. 
ALLEPUZ ROS, Ma TERESA. La gestión del capital intelectual: nuevos parámetros de análisis para la economía de la información. En: Jornadas Españolas de Documentación. FESABID 2000(7ª. Bilbao. 2000) ; p. 499-505

BARCELÓ LLAUGER, MARÍA. Escenarios de implantación para un proyecto de gestión del conocimiento. En: Jornadas prácticas de Gestión del conocimiento en las organizaciones $\left(2^{\circ}\right.$. Madrid. 2000)

BARRULAS, MARIA JOAQUINA. From Information Management to Knowledge Management in SMEs: is there a role for the librarian/documentalist? En: Infoum'99 p. 38-43

BUENO CAMPOS, EDUARDO. La gestión del conocimiento en la nueva economía. En: Jornadas prácticas de Gestión del conocimiento en las organizaciones ( $1^{\circ}$. Madrid. 1999)

BUENO CAMPOS, EDUARDO. De la sociedad de la información a la del conocimiento y el aprendizaje: La necesidad de programas de dirección del conocimiento y aprendizaje. En: Jornadas Españolas de Documentación. FESABID 2000 (7ª. Bilbao. 2000) ; p.647-657.

BUSTELO RUESTA, CARLOTA; CASTRO CRUZ, KATTIA. Implantación de un Sistema de Gestión de la Información: Superintendencia General de Valores de Costa Rica. En: Jornadas prácticas de Gestión del conocimiento en las organizaciones ( $1^{\circ}$. Madrid. 1999)

BUSTELO RUESTA, CARLOTA. Gestión documental en las empresas: una aproximación práctica. En: Jornadas Españolas de Documentación. FESABID 2000 (7ª. Bilbao. 2000) ; p. I 89-195.

CORNELLA, ALFONS. Gestió dels recursos d'informació: la informació com a actiu de l'empresa. En: Jornadas Catalanas de documentación (4. Barcelona. 1992)
DUFFY, JAN. Knowledge management: what every information professional should know. En: The information management journal, vol. 34, n. 3 (july 2000) ; p. 10-16

EDVINSSON, LEIF ; MALONE, MICHAEL S.. El capital intelectual. Barcelona: Gestión 2000, 1999

GARCÍA-MORALES HUIDOBRO, ELISA. Herramientas para la gestión del conocimiento: el Mapa Documental, una experiencia práctica de implantación sobre la Intranet corporativa. En: Jornadas prácticas de Gestión del conocimiento en las organizaciones $\left(2^{\circ}\right.$. Madrid. 2000)

HENCZEL, SUSAN. The information audit as the first step towards effective knowledge management. En: Knowmap, vol. I, n. I (2000)

HUANG, KUAN-TSAE. Calidad de la información y gestión del conocimiento. Madrid: AENOR, 2000

JERICÓ RODRÍGUEZ, PILAR. Capital intelectual. Concepto y conceptos: capital estructural, capital humano, capital clientes. En: Jornadas prácticas de Gestión del conocimiento en las organizaciones (1\%. Madrid. 1999)

ROGERS, EDWARD W. Why do we need to see our knowledge? En: Know Map, vol.I, n. I (2000)

SAFFADY, WILLIAM. Knowledge Management: an overview. En: The information management journal, vol. 34, n. 3 (july 2000) ; p. $4-8$

\section{Notas}

I. Leif Edvinsson ; Michael S. Malone. El capital intelectual. Barcelona: Gestión 2000, 1999

2. Algunos autores, como Edvinsson y Malone, lo incluyen como parte del capital estructural.
3. BUENO, Eduardo. La gestión del conocimiento en la nueva economía. En: Jornadas prácticas de Gestión del conocimiento en las organizaciones ( ${ }^{\circ}$. Madrid. 1999) ; p. I- I4. 